\title{
Current perspective on osteoarthritis: a narrative review
}

\author{
Sony Pokhrel', Ojashwi Nepal'
}

\section{Author(s) info:}

${ }^{1}$ School of Science, Kathmandu University, Kavre

${ }^{2}$ Department of Physiology, School of Medical Sciences, Kathmandu University, Chaukot, Kavre

\section{Correspondence:}

\section{Dr. Ojashwi Nepal, MBBS, $M D$}

Department of Physiology, Pre-Clinical Complex, School of Medical Sciences, Kathmandu University, Chaukot, Kavre

Email:

ojashwi@kusms.edu.np

DOI :

https://doi.org/10.3126/jpsn.v2i1.42587

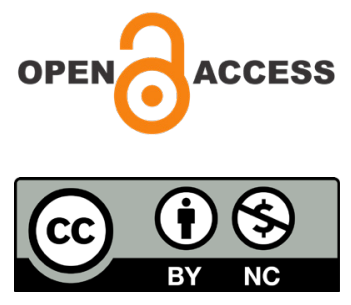

C JPSN

\begin{abstract}
Introduction: Osteoarthritis is the most prevalent chronic inflammatory joint disease affecting mobility in humans. It is caused by inflammation, breakdown, and eventual loss of cartilage in the joints. It is one of the ten most disabling diseases in developed countries. In this review we aimed to appraise the current evidence for the short as well as long term prevention and available effective treatment approaches for osteoarthritis. The objective of this study is to discuss about the risk factors, clinical methods of treatment, prevention and recent development in pathophysiological understanding of osteoarthritis.
\end{abstract}

Materials and methods: Full length published articles and journals were collected from different reliable sites, PubMed, Hinari and Medline. Among the accessed materials, 52 full length articles were selected excluding studies that only provided abstract as published papers.

Results: The incidence and prevalence was high among the people who had suffered from joint injury or undergone joint surgery recently in their lives. The risk was also high in people with bone malalignment, altered shape of bone and muscle strength. High muscle/fat ratio showed a protective effect against KOA prevalence. The incidence also depends on age and sex, obesity, bone density and mass, diet and genetic factors.

Conclusions: The risk is high among people with joint injury, old people, obese, people with poor diet, patients undergone joint surgery and in women after menopause. Definitive treatment for OA hasn't been available yet but, there are ways for prevention and treatment of OA. Pharmacological treatment includes NSAIDs, non-pharmacological includes exercise, physical activity and diet whereas surgical method includes total joint replacement.

Keywords: Bone malalignment, hip osteoarthritis, knee osteoarthritis 


\section{INTRODUCTION}

Osteoarthritis (OA) is the most prevalent long term chronic inflammatory joint disease, commonly affecting joints in the knees, hands, feet, hips, fingers, and/or lower spinal regions causing gradual loss of cartilage.[1] It is characterized by the deterioration of cartilage in joints which results in bones to rub together and creates stiffness, pain, and impaired movement.[2] Symptomatic $\mathrm{OA}$ is defined as the presence of radiographic OA plus symptoms including pain, aching, and not all individuals with radiographic OA present with symptomatic OA. The individual feels pain, stiffness and aching. Radiographic $\mathrm{OA}$ is diagnosed based on non uniform joint space lost, osteophyte formation, cyst formation and subchondral sclerosis on $\mathrm{x}$-ray. It is possible to have symptomatic OA without radiographic OA and vice versa. Post-traumatic OA develops after joint injury. Injury may be in the form of fracture, cartilage damage, acute ligament sprain, or chronic ligamentous instability (or a combination of these). Osteoarthritis is classified into two groups. $[3,4]$ Primary osteoarthritis can be localised or generalised, the latter more commonly found in postmenopausal women, with development of Heberden's nodes.[5] Secondary osteoarthritis has an underlying cause, such as trauma, obesity, Paget's disease, or inflammatory arthritis. [6] It is one of the ten most disabling diseases in developed countries. [11] Globally, the number of persons disabled by OA has more than doubled over the past 25 years. Increases in the prevalence and the metric of 'years lived with disability' from 140.5 per 10,000 and 7.3 million to 301.6 per 10,000 and 16.3 million were observed between 1990 and 2016, respectively.[3,4] In addition, there is emerging evidence that the prevalence of knee OA has doubled since the mid20th century. The 2010 Global Burden of Disease Study reports that the burden of musculoskeletal disorders is much larger than estimated in previous assessments and accounts for $6.8 \%$ of DALYs (Disability Adjusted Life Years) worldwide[12,13]. An estimated $10 \%$ to $15 \%$ of all adults aged over 60 have some degree of OA, with prevalence higher among women than men. The prevalence of OA is increasing due to population ageing and an increase in related factors such as obesity.[14]According to the United Nations, by 2050 people aged over 60 will account for more than $20 \%$ of the world's population by OA.[15]

During the development of OA, the metabolism and structure of the entire joint progressively changes, with damaged cartilage, remodeled subchondral bone and chronic inflammation of the synovium. $[7,8]$ These metabolic and structural changes to the joint adversely affect joint biomechanics which leads to pain and impaired weight-bearing activities, joint stiffness, limited ranges of motion and decreased physical function. [9] As there is limited capacity to repair and restore cartilage [10], at present there is no effective treatment for OA. Modern definitions of OA propose that it is a chronic low-grade inflammatory disease with gradually altering immune-metabolism of joints. $[14,17]$ It also suggests that increased oxidative stress contributes to the progression of OA.[18] In OA, exposure to inflammatory cytokines such as interleukin-1 $\beta$ (IL-1 $\beta$ ) increases oxidative stress by stimulating the production of reactive oxygen species (ROS) and nitric oxide (NO) in chondrocytes and synoviocytes, resulting in an imbalance of anabolic synthesis and catabolic breakdown of the cartilage matrix, leading to a loss of cartilage. $[14,18]$ Thus, strategies that lead to reductions in inflammatory cytokines and oxidative stress appear to slow the progression of $\mathrm{OA}$, indicating the potential of increasing intake of antioxidants for OA management.[19,20]

\section{Scientific investigations for development of diagnostic tools and effective treatments}

Osteoarthritis can be diagnosed by physical examination and, where necessary, with x-ray, MRI scan and arthroscopy. However, these diagnostic tools have low sensitivity and specificity.[15] There are no biomarkers for OA that can be used in clinical practice at this time. The treatment of $\mathrm{OA}$ involves treating the associated pain and inflammation; improve function and quality of life of patients by symptom-modifying pharmaceutical drugs (e.g., pain killers), lifestyle modifications (e.g., weight loss and exercise); viscosupplementation with intraarticular hyaluronate injections; intra-articular corticosteroid injections; joint replacement surgery.[16,17] The use of magnetic resonance imaging to detect the presence of cartilage and bone marrow lesions, osteophytes, and effusion is gaining popularity, though no standard magnetic resonance imaging-based definition of OA exists. Commonly, OA is graded radiographically using the Kellgren-Lawrence (K-L) scale.[7] The K-L scale ranges from 0 to 4 based on the presence and degree of osteophytes, joint-space narrowing, 
sclerosis, and deformity, with grades of 2 or higher indicating the presence of radiographic OA. The $\mathrm{K}-\mathrm{L}$ scale does not consider symptoms when defining OA severity. OA is basically related to ageing, but it is also associated with a variety of other both modifiable and non modifiable risk factors, including: obesity, lack of exercise, genetic predisposition, bone density, occupational injury, joint trauma and instability, gender, metabolic and endocrine diseases.

The Osteoarthritis Initiative (OAI) is a multi-center, longitudinal, prospective observational study of knee osteoarthritis (OA). The OAI maintains a natural history database for osteoarthritis that includes clinical evaluation data, radiological (x-ray and magnetic resonance) images, and a biospecimen repository from 4796 men and women ages 45-79 enrolled between February 2004 and May 2006. Four 3.0 Tesla MRI scanners, one at each clinical center, were dedicated to imaging the knees of OAI participants annually over four years. The seven-year project recruited participants who had, and those who are at high risk for developing, symptomatic knee osteoarthritis. All data and images collected are available to researchers worldwide to help quicken the pace of biomarker identification, scientific investigation and $\mathrm{OA}$ drug development. Access to biospecimens is by application to the National Institute of Arthritis, Musculoskeletal and Skin Diseases (NIAMS). [10]

\section{Risk factors of OA}

Approximately $30 \%-65 \%$ of the risk of osteoarthritis (OA) is genetically determined.[4, 5, 7]A recent review by Warner et al highlighted the main findings from genetic association studies on $\mathrm{OA}$ to date. They reported that genome-wide associated scan (GWAS) studies have so far identified 21 independent susceptibility loci for OA. Since this review's publication, the single nucleotide polymorphism (SNP) rs4238326 in the ALDH1A2 gene was linked with knee osteoarthritis (KOA) risk in a Chinese sample study. This is relevant, as genetic variants within the ALDH1A2 gene was only previously linked with hand OA in European populations.[37]Data from the Chingford study also found that the SNP rs11688000 in the neurokinin 1 receptor gene (TACR1) was associated with decreased risk of symptomatic OA. [27]

Injury/Surgery: Anterior cruciate ligament (ACL) injury, meniscal tear (MT), and direct articular cartilage damage following injury have all been linked to subsequent knee osteoarthritis (KOA) development namely post traumatic osteoarthritis (PTOA).[4,5,7,12] Three classes of chondral and osteochondral injuries can be identified based on the type of tissue damage and the repair response: damage to the joint surface that does not cause visible mechanical disruption of the articular surface, but does cause chondral damage and may cause subchondral bone injury; mechanical disruption of the articular surface limited to articular cartilage; and mechanical disruption of articular cartilage and subchondral bone.

People who sustain a knee injury are 4.2 times more likely to develop osteoarthritis (OA) than those without a history of knee injury.[12]

A systematic review by Luc et al showed that overall rates of OA development were higher in patients after ACL reconstruction (44\%) than in those who remained ACL deficient (37\%), with an odds ratio (OR) of 1.29 (95\% confidence interval [CI] $1 / 4$ 1.06, 1.52). Prevalence of PTOA was greater in ACL-reconstructed individuals through the first 2 decades after injury; however, PTOA prevalence was $34 \%$ greater in ACL-deficient compared with -reconstructed patients in the third decade after injury.[12] Stein et al reported radiographic changes in $19.2 \%$ of patients after medial meniscal repair compared with $60 \%$ of patients after partial medial meniscectomy at 8-year follow up. These data indicate a greater risk of PTOA development as the amount of meniscus that is removed increases. [12]

Joint loads/Alignment: In an OAI study, varus thrust (i.e., first appearance/ worsening of varus alignment during stance) was associated with KOA progression, but not KOA incidence. In the Multicenter Osteoarthritis Study (MOST), varus thrust increased the odds of worsening medial bone marrow lesions (BMLs) and medial cartilage loss as well as the odds of incident medial BMLs of the knee among those with KOA and those with increased risk of KOA, respectively. [17]

Bone/Joint Shape: Contributing to the body of evidence, a recent population-based OA cohort study in France used 5 measures to describe hip morphology. Among all measures, acetabular index was most strongly associated with the severity and progression of hip OA. In addition, the Rotterdam Study found that those with cam deformity or acetabular dysplasia had double 
the risk of developing hip OA compared to those without deformity. [18]

Muscle Strength: The association between muscle strength and $\mathrm{OA}$ may vary depending on the muscles and joints being studied. In an examination of anterior cruciate ligament (ACL) injured knees, high thigh muscle cross-sectional area (CSA) and high muscle/fat ratio had a protective effect against KOA prevalence. On the other hand, among OAI subjects without radiographic KOA and with minimal extension strength variability, higher total extensor CSA and vastusmedialis CSA were found to increase patellofemoral cartilage loss over time. There was also a strong positive association between extensor-flexor CSA ratio and patellofemoral cartilage deterioration. Similarly, higher knee extensor strength in adolescent men was associated with greater risk of KOA by middle age in a longitudinal study of Swedish registries. However, in a cross-sectional study of hip muscle strength and joints of subjects with hip OA, greater isometric strength of hip and thigh muscle groups was associated with better self-reported physical function. $[17,18]$

Age and Sex: The exact mechanism is not known, but is likely related to a combination of changes in the capacity for joint tissues to adapt to biomechanical insults, and age being a proxy for the accumulation of a sufficient set of risk factors over the years. There is seen a decline in OA at older ages. $[18,19]$

Female sex is associated with higher prevalence and greater severity of OA.[20] The increase in OA prevalence and incidence at the time of menopause has led to hypotheses regarding the role of estrogen in OA, such as the loss of estrogen potentially unmasking the symptoms of OA by enhancing pain sensitivity. However, results from observational studies and clinical trials have been conflicting regarding estrogen effects on $\mathrm{OA}$ and perhaps could be explained by other factors (reduced volume of cartilage, bone loss or lack of muscle strength).[21]

In the study of Korean population[20], prevalence progressively increased with age and was the highest in patients more than 80 years old (female: 63.3\%).In this study, the prevalence of symptomatic knee OA in males was $4.5 \%$. The female group showed a prevalence of $19 \%$ in the knee, which was significantly greater than in males.

Similar trends were seen in studies of Chinese
population[21].In studies of elderly Irish population[19] prevalence was higher for women and for those aged 80 years was more than twice that of those aged 50-60 years.

Obesity: Studies all over the world suggest the association of OA with obesity. [22] However, in study of risk factors for radiographic hand OA, no association was found between overweight/ obesity and hand OA in either cross-sectional or longitudinal analyses.[23] In a meta-analysis, those who were obese or overweight had 2.96 times higher risk of incident knee OA compared with those who were normal weight (95\% CI 2.563.43). Decreasing BMI by 2 units or more over 10 years $(\sim 5 \mathrm{~kg})$ was associated with $50 \%$ lower risk of developing symptomatic knee OA among women, 36 findings supported by a recent meta-analysis. [24] Previous studies reinforced the hypothesis showing the relation between metabolic syndrome and $O A$ [25] but recent studies didn't find significant association.[27] Though based on previous studies, association between obesity and hip OA had been weak, relatively recent cross-sectional study from Japan and a prospective cohort study from Spain found an independent association between weight gain and hip OA diagnosis. [26]

Bone Density and Mass: New evidence suggests that high systemic bone mineral density (BMD) predates early structural KOA features; higher spine and total hip BMD were recently linked to progression of tibio-femoral cartilage defects as measured by MRI in adults without clinical symptoms. [28]Contradicting this low BMD has been associated cross-sectionally with reduced joint space width at the hip, which could be a reflection of effects of existing OA. That is, once symptomatic OA has developed, an individual may decrease their physical activity and therefore loading of the joint, which in turn can contribute to low BMD. [29]

Vitamins/Diet: As vitamin D plays a major role in cartilage and bone metabolism, it has been hypothesized that low levels of it may increase OA risk.[30]After two years studies on test subjects, effusion synovitis (measured by MRI) remained stable in the vitamin $\mathrm{D}$ group but increased in the placebo group. Those with consistently sufficient 25-hydroxyvitamin D levels also had less loss of tibial cartilage volume, less increase in effusion synovitis, and less decrease in physical functioning compared to those with consistently insufficient levels. This supports the role of Vitamin D to 
prevent risks of OA. [31]

Findings from two prospective cohort studies also showed that higher total fiber intake was related to lower risk of symptomatic KOA, but its relation to radiographic KOA was unclear. [27, 32] Finally, higher adherence to a Mediterranean diet was associated with lower prevalence of clinical and radiographic KOA. [33, 34]

\section{Treatment}

\section{Pharmacological treatment}

Oral NSAIDs: A study published in the Lancet focused on effectiveness of different NSAIDs through a network meta-analysis of randomized controlled trials of any NSAID (1980-2015, including coxibs), paracetamol, and placebo with over 100 participants per group. They identified 74 trials with over 58,000 participants, and found that all NSAID preparations regardless of dose improved pain vs. placebo; no support for the effectiveness of paracetamol; [39] the greatest effect size for diclofenac and etoricoxib $(\sim 0.6)$, concluding that diclofenac $150 \mathrm{mg}$ per day is the most effective currently available NSAID for pain and function in OA. This meta-analysis did not consider safety outcomes, particularly cardiovascular risk, which has been noted to be similar between coxibs and diclofenac leading to reduced utilization of diclofenac in recent years. Thus, providers should consider the risk/benefit ratio of these therapies, and oral NSAIDs should be used at the lowest effective dose and for the shortest possible time. $[34,35]$

In a German study, overall, 63.4\% (95\% CI: $63.3 \%-63.5 \%$ ) of the studied patients had received prescriptions for analgesics and $44.1 \%$ for NSAIDs. [36]

Topical NSAIDs: Topical NSAIDs are an attractive option for OA management given their safety profile. The Cochrane Review of topical NSAIDs for musculoskeletal pain was updated to include more than 10,000 participants in 39 studies (all randomized, double-blind, placebocontrolled trials in adults with moderate to severe musculoskeletal pain and at least 10 subjects per arm). All included studies were of OA and were moderate to high quality. A study compared a novel topical NSAID, s-flurbiprofen plaster, to standard flurbiprofen commercially available in Japan in 633 individuals with knee $\mathrm{OA}$ and suggested a modest but significant benefit to the investigational drug; both were shown to be safe. [40, 41]

Intra-articular corticosteroid/Intra-articular hyaluronic acid: McCabe, et al., in their study found that all patients reported reduction in pain at 3-4 weeks post IASI. [42] Investigators from the OA Trial Bank performed an individual patient data meta-analysis of published randomized controlled trials of IASI in hip or knee OA by requesting data from corresponding authors of all eligible trials. [43] The authors found that IASI had significant short- ( $<4$ weeks $)$ and mid- (1-3 months) term benefits, but no effect on long term- (up to 12 months) outcomes, with no difference in signs of inflammation. $[44,45]$

Zhang, et al., considered the importance of aspiration of a joint prior to IAHA administration, randomizing 92 symptomatic knee OA patients to maximal aspiration and 88 to no aspiration prior to weekly IAHA for 5 weeks, with 25-week followup. The authors noted that visual analog scale (VAS) pain with walking and WOMAC function improved more in the aspiration group, but there was no difference in global "overall effectiveness" as rated by the patient or the investigator.[46]

A study by Shen et al found that PRP was superior to other intra-articular injections in terms of pain relief and function improvement through 3 to 12 months. Also, the risk of adverse events in PRPtreated participants was not significantly increased in comparison with other intra-articular injections. $[47,48]$

\section{Surgical treatment}

Total joint replacement: According to a study, the 2010 prevalence of total hip and total knee replacement in the total U.S. population was $0.83 \%$ and $1.52 \%$, respectively. Prevalence was higher among women than among men and increased with age, reaching $5.26 \%$ for total hip replacement and $10.38 \%$ for total knee replacement at eighty years. [48] These estimates corresponded to 2.5 million individuals (1.4 million women and 1.1 million men) with total hip replacement and 4.7 million individuals (3.0 million women and 1.7 million men) with total knee replacement in 2010. Secular trends indicated a substantial rise in prevalence over time and a shift to younger ages. Given the success of total hip and knee replacement in improving function and quality of life of 
individuals with severe arthritis, the current trends will likely continue in the coming decades. $[49,50]$

\section{Non-Pharmacological treatment}

Weight loss: An 18-week program out of Australia, OA Healthy Weight for Life, enrolled 1383 individuals with a mean age of 64 years and baseline BMI of $34 \mathrm{~kg} / \mathrm{m} 2$ ( $82 \%$ were obese). Almost all (94\%) of those enrolled lost at least $2.5 \%$ of their baseline weight, and $1 / 3$ lost more than $10 \%$. The researchers noted a dose-response relationship between change in KOOS (Knee Injury and Osteoarthritis Outcome Score) and percentage weight change, concluding that loss of at least $7.7 \%$ of baseline weight was needed to achieve a minimal clinically important difference in WOMAC function (derived from the KOOS).[51]

A study published in the Journal of the American Medical Association reported improvements in several OA-relevant measures in a cohort of 2200 people following bariatric surgery. The median pre-surgery BMI in this group was $46 \mathrm{~kg} / \mathrm{m} 2 ; 70 \%$ had 3-year follow up where the median weight loss was $30 \%$ of baseline, accompanied by significant improvements in knee and hip pain and function by WOMAC. The majority of these patients had clinically significant improvements in body pain, physical function, and walking capacity, although the percent of patients with improvement in pain decreased between one and three years postoperatively. [52]

Exercise/Physical activity: Exercise is an integral component of conservative nonpharmacological management of osteoarthritis and is advised by clinical guidelines for all patients, irrespective of disease severity, age, co-morbidity, pain severity, or disability (Conaghan et al 2008, Hochberg et al 2012, Zhang et al 2008). The studies vary particularly with regard to the type, dosage, mode of delivery, and duration of the exercise program. $[35,36]$

A recent study discovered an increase in IL10 levels of synovial fluid after acute resistance exercise in patients with OA of the knee. IL10 is an antiinflammatory cytokine with chondroprotective properties. It was shown that aquatic exercise was beneficial only in the beginning and in obese people whereas land-based aerobic and strengthening exercises had a continuous effect for as long as they were carried out. [52]
Mediterranean diet (MD) and other supplements: The findings of a systematic review on MD in relation to $\mathrm{OA}$ indicate positive associations between MD and improved quality of life in participants with OA. Biomarkers of inflammation and cartilage degradation related to OA were also analyzed and significant differences were detected for IL1- $\alpha$ ( $p=$ 0.019 ), which was lower in the diet group. However, the epidemiological evidence is limited, and longer interventions are required to evaluate the long-term efficacy of the MD for improving symptomatology and preventing OA. [37, 38]

A review demonstrates that the most widely used supplements (eg, glucosamine and chondroitin) are not clinically effective in reducing pain and improving physical function at short term in patients with OA. No supplements demonstrated clinically important effects for pain at medium term and long term except for green-lipped mussel extract and undenatured type II collagen, which suggested clinically meaningful effects on pain reduction at medium term. [51]

Contradicting this, a study by Zhu et al, suggests that oral chondroitin in recommended dosage is more effective than placebo on relieving pain and improving physical function. Compared with placebo, glucosamine showed significant effect on the outcome of stiffness. [52] Hence, further studies are necessary to come to a definitive conclusion.

\section{CONCLUSION}

Definitive treatment hasn't been found yet, but treatment of $\mathrm{OA}$ is available and is preventable. Pharmacological treatment includes NSAIDs, non-pharmacological includes exercise, physical activity and diet whereas surgical method includes total joint replacement. But, none of the mentioned treatment promises $100 \%$ cure of OA. Incidence and prevalence was high among people with joint problems; be it malalignment, shape of the bone, their density or a recent surgery. ACL injury, meniscal tear (MT), and direct articular cartilage damage was found to be linked to subsequent KOA development. The risk was high among old people and in women after menopause, people who are obese and the people with poor diet.

\section{CONFLICT OF INTEREST}

None. 


\section{REFERENCES}

1. M. Hiligsmann, et al., Health economics in the field of osteoarthritis: an expert's consensus paper from the European Society for Clinical and Economic Aspects of Osteoporosis and Osteoarthritis (ESCEO), Semin. Arthritis Rheum. 43 (3) (2013) 303-313.

2. T. Vos, et al., Global, regional, and national incidence, prevalence, and years lived with disability for 328 diseases and injuries for 195 countries, 1990-2016: a systematic analysis for the Global Burden of Disease Study 2016, Lancet 390 (10100) (2017) 1211-1259.

3. T. Vos, et al., Global, regional, and national incidence, prevalence, and years lived with disability for 301 acute and chronic diseases and injuries in 188 countries, 19902013: a systematic analysis for the Global Burden of Disease Study 2013, Lancet 386 (9995) (2015) 743-800.

4. I.J.Wallace, etal.,Knee osteoarthritis hasdoubledinprevalencesincethe mid-20th century, Proc. Natl. Acad. Sci. 114 (35) (2017) 9332-9336.

5. R.F. Loeser, Aging and osteoarthritis, Curr. Opin. Rheumatol. 23 (5) (2011) 492-496.

6. A.E. Wluka, C.B. Lombard, F.M. Cicuttini, Tackling obesity in knee osteoarthritis, Nat. Rev. Rheumatol. 9 (2012) 225.

7. D. Blalock, et al., Joint instability and osteoarthritis, Clin. Med. Insights Arthritis Musculoskelet. Disord. 8 (2015) CMAMD.S22147.

8. D.T. Felson, et al., The incidence and natural history of knee osteoarthritis in the elderly, the Framingham osteoarthritis study, Arthritis Rheum. 38 (10) (1995) 1500-1505.

9. T.D. Spector, A.J. MacGregor, Risk factors for osteoarthritis: genetics1 1Supported by Procter \& Gamble Pharmaceuticals, Mason, OH, Osteoarthr. Cartil. 12 (2004) 39-44.

10. Osteoarthritis Initiative (OAI) [Internet]. National Institute on Aging. 2021 [cited 5 January 2021]. Available from: https://www.nia.nih.gov/research/resource/ osteoarthritis-initiative-oai

11. Q. Zhuo, et al., Metabolic syndrome meets osteoarthritis, Nat. Rev. Rheumatol. 8 (2012) 729.

12. K. Louati, et al., Association between diabetes mellitus and osteoarthritis: systematic literature review and metaanalysis, RMD Open 1 (1) (2015).

13. S. Glyn-Jones, et al., Osteoarthritis, Lancet 386 (9991) (2015) 376-387.

14. A. Mobasheri, et al., The role of metabolism in the pathogenesis of osteoarthritis, Nat. Rev. Rheumatol. 13 (2017) 302.

15. D. Sanghi, et al., Is radiology a determinant of pain, stiffness, and functional disability in knee osteoarthritis? A cross-sectional study, J. Orthop. Sci. 16 (6) (2011) 719-
725

16. F. Guilak, Biomechanical factors in osteoarthritis, Best Pract. Res. Clin. Rheumatol. 25 (6) (2011) 815-823.

17. F. Berenbaum, Osteoarthritis as an inflammatory disease (osteoarthritis is not osteoarthrosis!), Osteoarthr. Cartil. 21 (1) (2013) 16-21.

18. A.K. Grover, S.E. Samson, Benefits of antioxidant supplements for knee osteoarthritis: rationale and reality, Nutr. J. 15 (1) (2016).

19. N. Ghoochani, et al., The effect of pomegranate juice on clinical signs, matrix metalloproteinases and antioxidant status in patients with knee osteoarthritis, J. Sci. Food Agric. 96 (13) (2016) 4377-4381.

20. Bannuru RR e. Therapeutic trajectory following intraarticular hyaluronic acid injection in knee osteoarthritis-meta-analysis. - PubMed - NCBI [Internet]. Ncbi.nlm. nih.gov. 2019 [cited 25 December 2019]. Available from: https://www.ncbi.nlm.nih.gov/pubmed/21443958

21. Park JH, HongJY, Han K, et al. Prevalence of symptomatic hip, knee, and spine osteoarthritis nationwide health survey analysis of an elderly Korean population. Medicine(Baltimore).2017;96(12):e6372.doi:10.1097/ MD.0000000000006372

22. Sun $X$, Zhen $X, H u X, L i Y, G u S, G u Y$ et al. Osteoarthritis in the Middle-Aged and Elderly in China: Prevalence and Influencing Factors. International Journal of Environmental Research and Public Health. 2019;16(23):4701.

23. French H, Galvin R, Horgan N, Kenny R. Prevalence and burden of osteoarthritis amongst older people in Ireland: findings from The Irish LongituDinal Study on Ageing (TILDA). 2020.

24. Neogi T, Zhang Y. Epidemiology of Osteoarthritis. Rheumatic Disease Clinics of North America. 2013;39(1):1-19.

25. Palazzo C, Nguyen C, Lefevre-Colau M, Rannou F, Poiraudeau S. Risk factors and burden of osteoarthritis. 2020.

26. MatID1 S, HasifJaafar M, TeckNgID C, Sockalingam $S$, Raja J, Kamaruzzaman S. Ethnic differences in the prevalence, socioeconomic and health related risk factors of knee pain and osteoarthritis symptoms in older Malaysians.

27. VinaaE,b and C, Kwoha K. Epidemiology of Osteoarthritis: Literature Update. 2019

28. Jrheum.org. 2020 [cited 11 November 2020]. Available

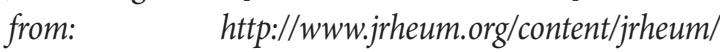
early/2017/07/11/jrheum.170026.full.pdf

29. PREVALENCE OF JOINT PAIN AND OSTEOARTHRITIS IN OBESE BRAZILIAN POPULATION | PACCA, Daniel Moreira; DE-CAMPOS, Gustavo Constantino; ZORZI, Alessandro Rozin; 
CHAIM, ElintonAdami; DE-MIRANDA, Jõao Batista | download [Internet]. De.booksc.org. 2020 [cited 11 November 2020]. Available from: https://de.booksc.org/ book/71425351/74527e

30. Reyes C, Leyland KM, Peat G, Cooper C, Arden NK, Prieto-Alhambra D. Association between overweight and obesity and risk of clinically diagnosed knee, hip, and hand osteoarthritis: a population-based cohort study. Arthritis Rheumatol. 2016; 68:1869-75. [PubMed: 27059260]

31. Dinu M, Pagliai G, Casini A, Sofi F. Mediterranean diet and multiple health outcomes: an umbrella review of metaanalyses of observational studies and randomised trials [Internet]. Semanticscholar.org. 2020 [cited 11 November 2020]. Available from: https://www.semanticscholar. org/paper/Mediterranean-diet-and-multiple-healthoutcomes\%3A-an-Dinu-Pagliai/981af3c36e6929a67aa59 $15 c e b 7 c f d 1902262658$

32. Morales I, Romera-Baures $M$, Roman-Viñas B and MajemL. Osteoarthritis and the Mediterranean Diet: A Systematic Review

33. Thomas A, Hubbard-Turner T, Wikstrom E, PalmieriSmith R. Epidemiology of Posttraumatic Osteoarthritis. 2020.

34. PMC E. Europe PMC [Internet]. Europepmc.org. 2020 [cited 11 November 2020]. Available from: https:// europepmc.org/article/MED/21188091

35. Bennell K. Physiotherapy management of hip osteoarthritis. 2020.

36. [Internet]. 2020 [cited 11 November 2020]. Availablefrom: https://www.researchgate.net/publication/321692330 Osteoarthritis_year_in_review_2017_Clinical

37. Liu X, Machado G, Eyles J, Ravi V, Hunter D. Dietary supplements for treating osteoarthritis: a systematic review and meta-analysis.

38. Zhu X, Sang L, Dandong W, Rong J and Liying Jiang L. Effectiveness and safety of glucosamine and chondroitin for the treatment of osteoarthritis: a meta-analysis of randomized controlled trials.

39. [Internet]. Ismrm.org. 2020 [cited 11 November 2020]. Available from: http://www.ismrm.org/10/program/ ELECTRONICPOSTER.doc

40. Postler A, Ramos A, Goronzy J, Günther K, Lange T, Schmitt J, Zink A, Hoffmann F. Prevalence and treatment of hip and knee osteoarthritis in people aged 60 years or older in Germany: an analysis based on health insurance claims data.

41. HilalS, Kremers M, Dirk R. Larson D, Cynthia S, Walter K.Raynard E et al. Prevalence of Total Hip and Knee Replacement in the United States.

42. Kwong C, Woodmass J, Gusnowski E, Bois A, Leblanc $J$, More $K$ et al. Platelet Rich Plasma in Patients with Partial Thickness Rotator Cuff Tears or Tendinopathy Leads to Significantly Improved Short-Term Pain Relief and Function Compared to Corticosteroid Injection: A Double-blind Randomized Controlled Trial. 2020.

43. Moon D, Park Y, Song S, Kim M, Park J, Nam D, Kim D et al. Common Upper Extremity Disorders and Function Affect Upper Extremity-Related Quality of Life: A Community-Based Sample from Rural Areas.

44. Mannoni A, Briganti M, Bari M, Ferrucci L, Costanzo $L$, Serni $U$ et al. Epidemiological profile of symptomatic osteoarthritis in older adults: a population based study in Dicomano, Italy.

45. Kiadaliri A, Lohmander S, Moradi-lakeh M, Petersson I. High and rising burden of hip and knee osteoarthritis in the Nordic region, 1990-2015

46. Wang $Q$, Wang $T$, Xiao-feng $Q$, Yao $M$, Cui X, Wang Y, Liang Q. Manual Therapy for Hip Osteoarthritis: A Systematic Review and Meta-analysis.

47. BaeY, Shin J, Lee J, Kim M, Park K, Cho J, Ha I. Association between Hypertension and the Prevalence of Low Back Pain and Osteoarthritis in Koreans: A CrossSectional Study.

48. Wilder F, Barrett M, Farina E. Brief report on Jointspecific prevalence of osteoarthritis of the hand.

49. Kang X, Fransen M, Zhang Y, Li H, Ke Y, Lu M et al. The High Prevalence of Knee Osteoarthritis in A Rural Chinese Population: The Wuchuan Osteoarthritis Study.

50. Cameron K, Driban J, Svoboda S. Osteoarthritis and the Tactical Athlete: A Systematic Review.

51. Park JH, Hong JY, Han K, et al. Prevalence of symptomatic hip, knee, and spine osteoarthritis nationwide health survey analysis of an elderly Korean population. Medicine(Baltimore).2017;96(12):e6372.doi:10.1097/ MD.0000000000006372

52. Englund M. The role of the meniscus in osteoarthritis genesis. Med Clin North Am. 2009;93:37-43 